\title{
Citrobacter Koseri osteomyelitis of maxilla- A Rarity
}

\author{
Authors \\ Dr Payal Nanwani, Dr Vrinda Kolte, Dr Ramakrishna Shenoi \\ VSPM Dental College \\ Email:dr.payalpeshwani14@gmail.com \\ Mobile: 9994901097
}

\section{Introduction}

Citrobacter is gram-negative aerobic bacilli belonging to Enterobacteriaceae. C. diversus (C. koseri), C. amalonaticus and C. freundii are three major species in the genus. Citrobacter species mostly causes urinary tract and gastrointestinal infections. ${ }^{1}$ These usually affect immunocompromised hosts, geriatric patients, neonates as well as cause nosocomial infections. ${ }^{2}$ Citrobacter koseri (diversus) causes meningitis and brain abscess besides sepsis in neonates. ${ }^{3}$

Citrobacter osteomyelitis is an uncommon condition. There are only few septic arthritis and osteomyelitis cases reported due to Citrobacter species. ${ }^{4,5}$ Citrobacter Osteomyelitis in the geriatric patients often results from direct invasion of soft tissue infection because of vascular compromise such as diabetes mellitus and bone cultures may yield mixed microorganisms. ${ }^{4,5}$ Bone cultures should be evaluated to detect probable pathogens and determine proper antimicrobial therapy. $^{2}$

Osteomyelitis of maxilla was originally described by Rees in $1847 .{ }^{6}$ Osteomyelitis of facial bones is unusual condition. Osteomyelitis of maxilla is uncommon as compared to that of mandible because of extensive blood supply and strut like bone of maxilla makes it less prone to chronic infection. ${ }^{1,7}$

Here we report a case of Citrobacter koseri osteomyelitis in an elderly diabetic patient affecting maxilla. The concerned literature is reviewed and discussed.

\section{Case Report}

A 52-years-old man was referred to our outpatient department with his chief complain of regurgitation of fluid from both the nostrils since 4 months while gargling. Patient was known case of diabetes mellitus and hypertension since 5 years but due to lack of compliance was under medication only since 2 months. There was a history of liver cirrhosis 6-7 years back and was hospitalized for the same.

The patient gave a history of pain and mobility of teeth in upper right and left posterior region of jaw for which he underwent extraction of teeth in private clinic four months back. On clinical examination there were healed sockets of extracted teeth, however; in 14,15 and 27 region there were fistulus opening. (Figure 1) There was no pus discharge noted. 


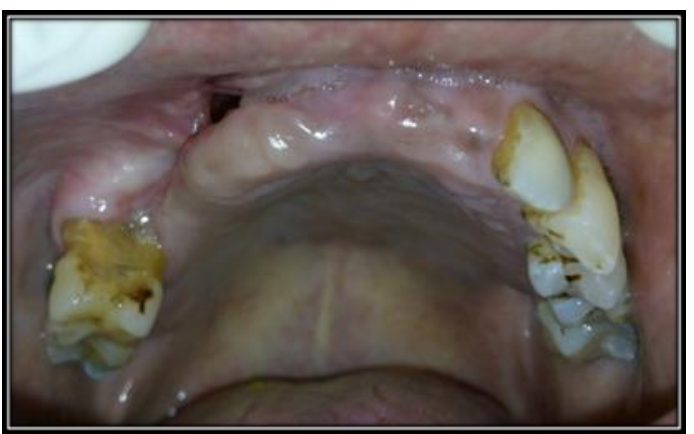

Figure-1: Oroantral fistula with 14, 15 region of maxilla

Routine laboratory investigations revealed changes of nephropathy with elevated blood urea $(64 \mathrm{mg} / \mathrm{dl})$ and creatinine $(1.8 \mathrm{mg} / \mathrm{dl})$ levels. He was also showing signs of uncontrolled diabetes (Fasting Blood Glucose- $153.6 \mathrm{mg} / \mathrm{dl} ; \mathrm{HbA}_{1} \mathrm{c}-$ $7.8 \%)$. Swab was obtained and sent for culture and identified as Citrobacter koseri by various biochemical tests and confirmed by VITEK-2 Compact automated system. Sensitivity demonstrated Citrobacter koseri species sensitive to amoxyclav, cefotaxime, ciprofloxacin, norfloxacin, gentamicin, imipenem and meropenem. Incisional biopsy was suggestive of necrotic bony tissue. Plain radiograph of paranasal sinuses showed bilateral maxillary sinusitis with bony erosionnoted on right side. The CT (MDCT16 slice Toshiba, Activion) report revealed areas of bone destruction in maxilla involving alveolar process and hard palate leading to oro-antral fistula in the region of premolars on right side and in the region of molars on left side. (Figure 2) Hard palate also showed sclerotic areas. Evidence of sequestrum was noted in the alveolar process on right side.

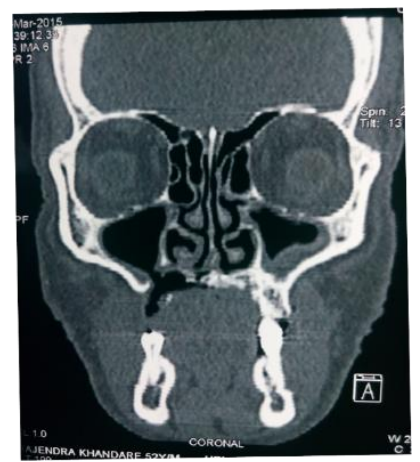

Figure-2: CT face: coronal section showing osteomyelitis involving right maxilla
The patient's diabetic status was controlled. He was posted under general anaesthesia for complete surgical excision (sequestrectomy) followed by closure of oro-antral fistula with local flaps. Posterior teeth were extracted from both sides of maxilla. The lining of oroantral fistula was excised followed by sequestrectomy on right side of maxilla. The lining of right maxillary sinus was exposed and removed followed by closure of the surgical site with local flap. Similar procedure was repeated on left side of maxilla, however the sinus lining was not exposed.

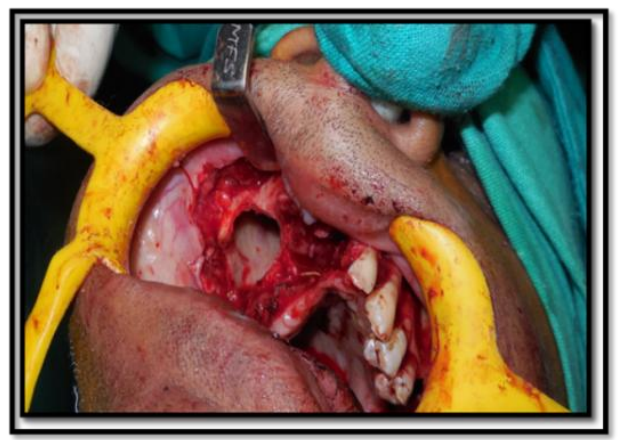

Figure-3: Intra-oral removal of the sequestrum and lining of right maxillary sinus

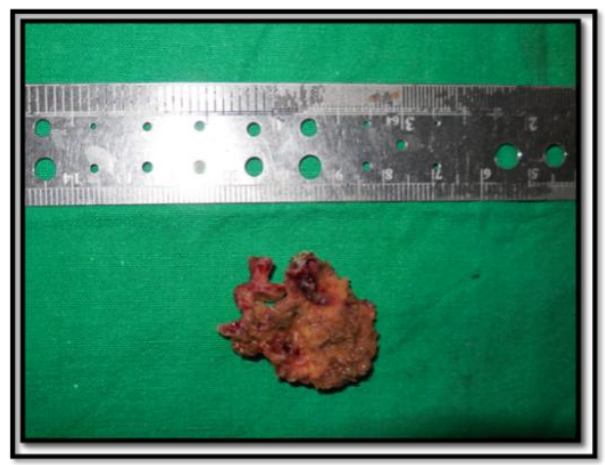

Figure-4: Dimensional view of the excised specimen of part of right maxilla

Excellent results were obtained with complete surgical excision and appropriate antibiotics and strict glycemic control. Antibiotic treatment was administered for 3 weeks. The wound healed uneventfully and laboratory findings returned to normal levels. The patient was discharged to continue with per-oral therapy with ciprofloxacin for another three weeks and the total treatment was completed in 6 weeks. Patient was on follow up for 6 months and there was no relapse. 


\section{Discussion}

Our case is one of the very uncommon cases of Citrobacter koseri (diversus) osteomyelitis of maxilla. Osteomyelitis due to Citrobacter koseri has been reported in two diabetic geriatrics in one paper which is a retrospective study conducted in 1980, evaluating Citrobacter infections over six year period. ${ }^{5}$ There has been another case reported involving a child with osteomyelitis of maxilla that led to meningitis by direct extension. ${ }^{8}$ One more case, 68-years-old man with diabetes mellitus was reported in 2011 who had 1st metatarsal osteomyelitis due to Citrobacter koseri. Citrobacter koseri osteomyelitis of maxilla in our patient probably resulted from direct spread of tissue infection because he had diabetes mellitus as predisposing factor besides his age.

The treatment of osteomyelitis varies from simple non-invasive approach to more invasive radical treatment. $^{7}$ Treatment can be conservative resection of affected bone with adequate clearance in all cases except in cases of osteomyelitis due to osteoradionecrosis (ORN) where resection has to be more radical. ${ }^{9}$ Wide spectrum antibiotics should be initially given, as osteomyelitis is usually present in association with an ulcer and soft tissue infection. Antibiotics like sodium fusidate, rifampicin, clindamycin and ciprofloxacin have good bone penetration. ${ }^{10}$ Finally, proper antibiotics should be administered based on microbiological examination of bone cultures.

\section{Conclusion}

An infection of Citrobacter koseri presenting as maxillary osteomyelitis has been a rarity in the reported literature. Citrobacter koseri can be a cause of aggressive bone destruction in osteomyelitis in debilitated, immunosuppressed or elderly patients; which can be fatal by spreading to base of the skull causing meningitis. The successful management of this condition involves timely diagnosis and the intervention involving a two pronged approach of surgical intervention in the form of sequestrectomy and culture guided antibiotic administration.

\section{Conflicts of Interest: Nil}

\section{References}

1. Karthik Shamanna, Rasika Rao, Asima Banu. Osteomyelitis of Maxilla: A Rare Case. Journal of Public Health Medicine Research 2014; 2(1):50-2.

2. Server Yagcia, Kader Arslana, Zeliha Kocak Tufana, Sami Kiniklia, Ali Pekcan Demiroza. Osteomyelitis Due to Citrobacter koseri Infection in a Diabetic Patient. Journal of Clinical Endocrinology and Metabolism 2011; 1(3):146-48.

3. Murray PR. Enterobacteriaceae. In: P.R. Murray, editor. Medical microbiology, Philadelphia: Mosby Elsevier; 2009. p. 301-14.

4. Kleint W, Herwig H. Septic osteomyelitis caused by Citrobacter with a contribution to the problem of cortisone administration in bacterial infections. Arztl Wochensch 1958; 13(43):965-69.

5. Jansen RD, Meadow WL, Schwartz IK, Ogata ES. "Bacteriological bit": Citrobacter diversus osteomyelitis in a neonate. Clinical Pediatrics (Phila) 1981; 20(12):791.

6. S P Lumba, A Nirola, B S Grewal. Healed osteomyelitis of Maxilla with tooth in the floor of nose. The Journal of Laryngology \& Otology Aug 1971; 85[08]:877-9.

7. Manimaran K, Suresh Kannan P, Kannan R. Osteomyelitis of maxilla bilateral involvement A case report. Journal of Indian Academy of Dental Specialists April-June 2011; 2[2]:57-8.

8. Wong SK, Wilhelmus KR. Infantile maxillary osteomyelitis with cerebral abscess. Journal of Pediatric Ophthalmology and Strabismus. 1986; 23(3):153-54. 
9. Prasad K C, Prasad S C, Mouli N, Agarwal S. Osteomyelitis in the head and neck. ActaOtolaryngologica2007; 127:194-205.

10. Edmonds M. The treatment of diabetic foot infections: focus on ertapenem. Journal of Vascular Health and Risk Management 2009; 5:949-63. 